SHORT REPORT

\title{
From categorical diagnosis to dimensional assessment of borderline personality
}

\author{
Izabela Huczewska ${ }^{A, B, C, D, E, F}$, Patrycja Didyk $k^{B, C, E, F}$, Radosław Rogoza (D) ${ }^{A, D, E, G}$ \\ Cardinal Stefan Wyszyński University in Warsaw, Warsaw, Poland
}

Recent research suggested that personality disorders could be diagnosed as a continuous phenomenon. Therefore, in our study we examined whether the dimensional model of pathological personality traits could be applied to the assessment of borderline personality ${ }^{1}$. For this purpose, we modified an existing measure of borderline personality, the McLean Screening Instrument for Borderline Personality Disorder (MSI-BPD), and scrutinized its psychometric properties (i.e., reliability, factorial structure, criterion validity). To assess criterion validity we calculated correlations with pathological personality traits. Our sample comprised 354 participants (67.8\% women). Confirmatory factor analysis revealed that the modified MSI-BPD mea- sures borderline personality as a global construct, giving one-factor structure. The reliability of the measurement was excellent $(\alpha=.90)$. Moreover, we found positive associations between borderline personality and all five pathological personality traits, which supports the validity of the continuous assessment of borderline personality. Our findings suggest that the DSM-5 dimensional model may be applied in the assessment of borderline personality.

\section{KEY WORDS}

borderline personality; DSM-5; dimensional model of borderline personality; PID-5

CORRESPONDING AUthor - Izabela Huczewska, Cardinal Stefan Wyszyński University in Warsaw, 5 Dewajtis Str., 01-815 Warsaw, Poland, e-mail: izabelahuczewska@gmail.com

AUthors' CONTRIBUtION - A: Study design - B: Data collection - C: Statistical analysis - D: Data interpretation .

E: Manuscript preparation · F: Literature search · G: Funds collection

to Cite this ARTicle - Huczewska, I., Didyk, P., \& Rogoza, R. (2019). From categorical diagnosis to dimensional

assessment of borderline personality. Current Issues in Personality Psychology, 7(4), 355-360.

RECEIVED 26.05.2019 · REVIEWED 03.09.2019 · ACCEPTED 04.10.2019 • PUBLISHED 12.11.2019 


\section{BACKGROUND}

The previous method of diagnosing personality disorders relied on a categorical system. This approach, used in the Diagnostic and Statistical Manual of Mental Disorders - DSM-IV-TR (APA, 2000), specifies personality disorders as distinct clinical entities, qualitatively different from each other and from normal personality (Strus et al., 2017). Accordingly, categorical diagnosis requires clinicians to provide a discrete answer, whether the person has the disorder or not. Although this approach has some pragmatic advantages (e.g., treatment decisions are also categorical), several limitations of such a paradigm need to be acknowledged. Firstly, multiple studies have documented excessive diagnostic co-occurrence of different personality disorders in the same patient (Trull, Scheiderer, \& Tomko, 2012). In fact, most of the individuals who meet the criteria for one personality disorder also fulfil the criteria for another. For instance, Tomko, Trull, Wood, and Sher (2014) reported that approximately half of borderline patients have at least one other personality disorder and found the highest rates of comorbidity with narcissistic, schizotypal, and dependent personality disorders. One explanation for these results is that many diagnostic categories in classification systems substantially overlap. The distinction between normal and aberrant personality is arbitrary and marked by unstable thresholds. Moreover, it is probable that two patients with the same diagnosis will not have any diagnostic features in common due to heterogeneity within personality disorders (Widiger \& Trull, 2007). For example, there are 256 combinations to meet diagnostic criteria for borderline personality (BP), and within each person, every criterion may vary in its degree of severity. Thus, many clinicians find it difficult to properly establish the diagnosis of BP (Biskin \& Paris, 2012).

\section{DSM-5 PATHOLOGICAL PERSONALITY TRAITS - A SHIFT TOWARDS DIMENSIONAL ASSESSMENT OF PERSONALITY DISORDERS}

The model of pathological personality traits was proposed in DSM-5 (APA, 2013) as an element of the hybrid system that combines dimensional and categorical approaches. In DSM-5, an individual's personality dysfunction is specified using a set of pathological traits, hierarchically organized into the five main domains: negative affectivity (e.g., anxiousness, emotional lability), detachment (e.g., withdrawal, intimacy avoidance), antagonism (e.g., callousness, deceitfulness), disinhibition (e.g., impulsivity, irresponsibility) and psychoticism (e.g., eccentricity, unusual beliefs and experiences). These dimensions represent an instantiation of the five-factor model (FFM) of personality, which hitherto has been the dominant model in the dimensional approach (Strus et al., 2017). The FFM enables a comprehensive, precise, and individualized depiction of one's personality structure within five basic personality dimensions (i.e., neuroticism, extraversion, openness, agreeableness, and conscientiousness). However, despite its usefulness in describing adaptive and dysfunctional facets of personality, the FFM encounters difficulties in capturing unique characteristics of personality disorders (Strus et al., 2017). Thus, the model of pathological personality traits extends the FFM to extreme and maladaptive counterparts of basic personality traits.

The predominance of this model over the categorical approach stems from its solid scientific foundations. While there is no empirical evidence that personality disorders are categorical (Hopwood et al., 2018), the dimensional approach is a researchdriven field, verified by genetic and neuropsychological studies (Esbec \& Echeburúa, 2015). Such a paradigm is a step towards making clinical psychology a truly evidence-based science (Stoyanov, Machamer, \& Schaffner, 2012).

\section{CATEGORICAL VERSUS DIMENSIONAL ASSESSMENT OF BORDERLINE PERSONALITY}

Borderline personality is characterized as an ongoing pattern of instability regarding interpersonal relationships, self-image and affect, typically marked by episodes of impulsivity (APA, 2013). In their everyday life, individuals with BP shift between extreme and easily triggered feelings of depression and anger (Biskin \& Paris, 2012). They often engage in potentially dangerous behaviours (e.g., substance abuse, reckless driving) and various forms of selfaggression (e.g., self-mutilation, suicide attempts, gestures or threats; APA, 2013). Interpersonally, individuals with BP are highly sensitive to any signs of rejection and perceive it even when it is not intended (Gunderson, Herpertz, Skodol, Torgersen, \& Zanarini, 2018). This vulnerability intertwines with neediness and desperate efforts to avoid being abandoned. Simultaneously, individuals with BP are in constant fear of dependency and losing autonomy. As a result, they often establish dysfunctional and debilitating relationships, alternating between over-involvement and withdrawal (APA, 2013). Finally, BP is associated with the lack of a coherent and stable sense of self which manifests primarily in chronic feelings of emptiness and uncertainty regarding one's actions, beliefs, values systems and goals (Biskin \& Paris, 2012).

The question whether BP should be assessed in a categorical or dimensional manner is of great 
importance, as BP remains one of the most prevalent and severe personality disorders (Gunderson et al., 2018). To date, several studies have empirically determined that the conceptualization of $\mathrm{BP}$ as a dimensional variable is more accurate than arbitrary categorization of individuals as borderline or non-borderline (Trull, Distel, \& Carpenter, 2011). Dimensional representations of personality pathology underpin the understanding of symptoms' heterogeneity, offer the lack of discrete boundaries between disorders and still give an important information about the sub-threshold traits of personality disorders (Trull \& Durret, 2005). In recent years, there has been an increasing interest in transition to dimensional assessment of personality disorders (Hopwood et al., 2018). However, despite the welldocumented limitations of the categorical approach and notable advantages of the dimensional paradigm, the diagnosis of BP still remains categorical and there is very little agreement on the applicability of a dimensional model for its assessment. A few studies (e.g., Bach \& Sellbom, 2016) that investigated relationships between BP and pathological personality traits, although finding them significant and positive, also revealed different patterns regarding the strength of these relations. Giving the existing uncertainty, our aim was to examine BP in the context of pathological personality traits.

\section{CURRENT STUDY}

In the light of foregoing literature, the dimensional understanding of BP is the preferable one. Hence, in our study we aimed to test the dimensional approach to BP. For this purpose, we modified a popular measure of BP, the McLean Screening Instrument for Borderline Personality Disorder (MSI-BPD; Zanarini et al., 2003), by replacing the dichotomous response scale with a 5-point Likerttype scale. Then, we analyzed its psychometric properties (i.e., factorial structure, reliability, and external validity).

First (H1), we hypothesized that the modified MSI-BPD would measure BP as a global construct, giving one-factor structure. Second, we expected (H2) the modified MSI-BPD to be a reliable measure of BP. Finally, we hypothesized (H3) that the modified version of MSI-BPD would be externally valid, which was examined through bivariate correlations with pathological personality traits. Based on previous studies (e.g., Bach \& Sellbom, 2016) we assumed that BP would be related mostly to high negative affect, but also positively associated with all five pathological trait domains. All of the data and used scripts are available at https:// osf.io/um47y/?view_only=524d5d18a4ac462483af1 ac4a68ac16f.

\section{PARTICIPANTS AND PROCEDURE}

\section{PARTICIPANTS}

Participants were 391 adults from the United States aged from 18 to 46 years. To detect invalid responses, we employed instructional manipulation checks (e.g., "If you are reading this sentence, please mark two") and excluded participants who responded incorrectly. The final sample comprised 345 participants (67.8\% women), aged from 18 to 46 years $\left(M_{\text {age }}=30.50\right.$, $\left.S D_{\text {age }}=7.56\right)$. Ethnicities included Caucasian (61.4\%), Black or African American (12.5\%), Hispanic or Latino (10.4\%), Asian or Pacific Islander (8.1\%) and $\mathrm{Na}-$ tive American or American Indian (0.6\%). Of all participants, $49 \%$ were single and $46.1 \%$ were married/ in a domestic relationship. Also, the majority of respondents had either graduated from college (31.2\%) or started college but had not yet completed their degree (31.9\%). Other respondents reported high school graduation $(15.4 \%)$ or vocational training $(7.8 \%)$ as their highest level of education, and $9 \%$ of participants had obtained a post-graduate degree.

\section{PROCEDURE}

The data were gathered via Clickworker: participants were recruited online and completed a larger set of self-report measures. All respondents were ensured confidentiality and compensated $\$ 0.60$ for completion of the study.

\section{MEASURES}

The McLean Screening Instrument for Borderline Personality Disorder. To assess BP, we used the McLean Screening Instrument for Borderline Personality Disorder (MSI-BPD; Zanarini et al., 2003). MSI-BPD is a 10-item, dichotomous (yes/no), self-report questionnaire (sample item: "Have you chronically felt empty?") that targets the nine DSM-IV criteria for borderline personality disorder. According to Zanarini et al. (2003) seven or more symptoms must be present to regard the screen as positive. In our study, we used the continuous 5-point Likert-type scale ranging from 1 (definitely no) to 5 (definitely yes).

Personality Inventory for DSM-5 Brief Form. Pathological personality traits were measured using the 25-item Personality Inventory for DSM-5 Brief Form (PID-5-BF; Krueger, Derringer, Markon, Watson, \& Skodol, 2013). PID-5-BF is a self-rating questionnaire designed to assess five personality trait domains: negative affect, detachment, antagonism, disinhibition, and psychoticism. Respondents answered on a 4-point scale ranging from 0 (very false or often false) to 4 (very true or often true). Each 
trait domain was measured by five items, for example "I get emotional easily, often for very little reason" (negative affect). A higher score indicated greater dysfunction in the specific domain.

\section{STATISTICAL ANALYSES}

To test the factorial structure of MSI-BPD we ran confirmatory factor analysis (CFA) using the weighted least squares means and variance adjusted (WLS$\mathrm{MV}$ ) estimator. The model fit was assessed using the comparative fit index (CFI) and the weighted root mean square residual (WRMR; Muthén, 1998-2004), which is found superior to the typically used root mean square error of approximation (RMSEA) in models with low degrees of freedom (Kenny, Kaniskan, \& MacCoach, 2015). The model may be deemed as well fitted to the data when the value of CFI is $\geq .95$ (Schermelleh-Engel, Moosbrugger, \& Muller, 2003) and WRMR $\leq 1$ (DiStefano, Liu, Jiang, \& Shi, 2017). To test the reliability of the measure - in addition to reporting the Cronbach's $\alpha$ - we calculated reliability estimates using the framework of the structural equation modelling as suggested by Saris and Gallhofer (2007) in order to control for the measurement error. That is, using Pearson's correlation coefficient we compared the estimated latent means to the summated score of all test items. A value close to 1 means that the measurement error has little influence on the measure true score.

\section{RESULTS}

\section{FACTORIAL STRUCTURE OF THE MSI-BPD}

Firstly, we tested the measurement model in which one latent factor of BP was loaded by all test items.
The results of the analyzed model $\left(\chi^{2}(35)=263.93\right.$, $p<.001$, CFI $=.952$, WRMR $=1.30)$ were ambiguous - the values of CFI suggested a good fit to the data, while the values of WRMR suggested some misspecifications. The inspection of modification indices revealed substantial correlations between three pairs of residual variances. The introduced changes yielded a good model fit $\left(\chi^{2}(32)=165.57, p<.001, \mathrm{CFI}=.972\right.$, WRMR $=0.98)$ and the factor loadings are presented in Figure 1.

The strength of the factor loadings was adequate (i.e., > .50, and with the exception of items 1 and $9>.70)$. In view of introduced modifications, the first hypothesis was confirmed, but the dimensional MSI-BPD measurement model could potentially be further improved.

\section{RELIABILITY OF THE MEASUREMENT}

The reliability of the measurement assessed by Cronbach's formula $(\alpha=.90)$ supported the hypothesis of the MSI-BPD quality. The estimated latent mean correlated significantly with the summated score at $r=.98(p<.001)$, which suggests that the influence of the measurement error on the true score is marginal. Thus, the second hypothesis, claiming that BP could be reliably measured using the modified MSI-BPD, was confirmed.

\section{CRITERION VALIDITY}

We analyzed the external validity of the modified MSI-BPD by calculating the correlations between the total score of the MSI-BPD and pathological personality traits. These results, as well as descriptive statistics for all studied variables (i.e., $M, S D$, and Cronbach's $\alpha$ ), are presented in Table 1 .

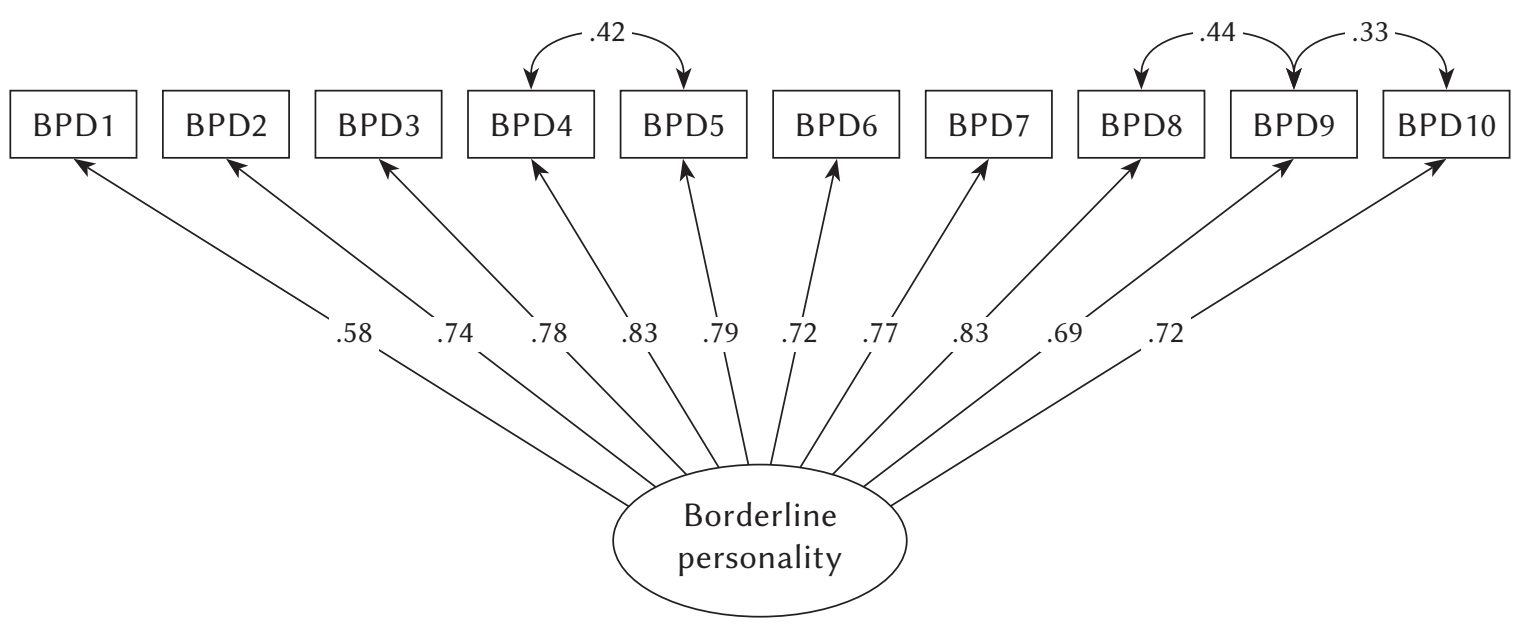

Figure 1. Measurement model of the MSI-BPD. 
Table 1

Bivariate correlations and descriptive statistics for all studied variables

\begin{tabular}{lcccc}
\hline Variable & Borderline personality & $M$ & $S D$ & $\alpha$ \\
\hline Borderline personality & 1 & 2.71 & 1.07 & .90 \\
Negative affect & $.68^{*}$ & 1.35 & 0.79 & .79 \\
Detachment & $.48^{*}$ & 0.98 & 0.70 & .72 \\
Antagonism & $.37^{*}$ & 0.47 & 0.52 & .71 \\
Disinhibition & $.41^{*}$ & 0.75 & 0.71 & .82 \\
Psychoticism & $.61^{*}$ & 1.00 & 0.64 & .71 \\
\hline
\end{tabular}

Note. ${ }^{*} p<.01$ (2-tailed).

As hypothesized, BP was positively related to all five pathological personality traits: it was strongly associated with negative affect and psychoticism, and moderately associated with detachment, disinhibition and antagonism.

\section{DISCUSSION}

This study aimed to test the applicability of the pathological personality traits model for BP assessment. For this purpose, we modified the existing version of MSI-BPD and scrutinized its psychometric properties. Our results supported the one-factor solution for the modified MSI-BPD and showed that it is a reliable measure of BP. Further, we found that BP is meaningfully associated with all five pathological personality traits (strongly with negative affect and psychoticism), which suggests that the modified MSI-BPD retained its criterion validity. These correlations seem to be in accordance with clinical descriptions of BP. For example, high psychoticism may cover cognitive difficulties in borderline patients (e.g., paranoid thoughts, stressrelated psychotic experiences; Biskin \& Paris, 2012), whereas negative affect corresponds with typical for BP emotional problems (e.g., easily triggered negative emotions, hypersensitivity to any signs of rejection).

The dimensional approach addresses many limitations of the categorical diagnosis (Trull \& Durret, 2005), enabling more complex, precise, and individualized assessment of the patient's psychopathology. Our findings suggest that the dimensional assessment of BP is empirically plausible. However, the results should be interpreted with caution as we relied on self-report measures that may be affected by various biases (e.g., self-presentation, introspective ability, or interpretation of particular items). Moreover, we employed online research methodology that may encounter problems regarding the credibility of information provided by respondents (e.g., age, gender). Thus, future studies with more sophisticated methodology are needed.

\section{ACKNOWLEDGEMENTS}

This work was supported by National Science Centre, Poland (2015/19/N/HS6/00685).

\section{ENDNOTE}

1 Throughout the paper, instead of borderline personality disorder, we use the term borderline personality as it is more applicable to dimensional understanding of personality pathology.

\section{RefERENCES}

American Psychiatric Association (2000). Diagnostic and statistical manual of mental disorders (4th ed., text rev.). Washington, DC: American Psychiatric Association.

American Psychiatric Association (2013). Diagnostic and statistical manual of mental disorders (5th ed.). Washington, DC: American Psychiatric Association.

Bach, B., \& Sellbom, M. (2016). Continuity between DSM-5 categorical criteria and traits criteria for borderline personality disorder. The Canadian Journal of Psychiatry, 61, 489-494. https://doi. org/10.1177/0706743716640756

Biskin, R., \& Paris, J. (2012). Diagnosing borderline personality disorder. Canadian Medical Association Journal, 184, 1789-1794. https://doi.org/10.1503/ cmaj.090618

Distefano, C., Liu, J., Jiang, N., \& Shi, D. (2017). Examination of the weighted root mean square residual: Evidence for trustworthiness? Structural Equation Modeling: A Multidisciplinary Journal, 25, 453-466. https://doi.org/10.1080/10705511.2017.13 90394

Esbec, E., \& Echeburúa, E. (2015). The hybrid model for the classification of personality disorders in DSM-5: A critical analysis. Actas Españolas de Psiquiatría, 43, 177-186. 
Gunderson, J. G., Herpertz, S. C., Skodol, A. E., Torgersen, S., \& Zanarini, M. C. (2018). Borderline personality disorder. Nature Reviews. Disease Primers, 4, 18029. https://doi.org/10.1038/nrdp.2018.29

Hopwood, C. J., Kotov, R., Krueger, R. F., Watson, D., Widiger, T. A., Althoff, R. R., ... \& Zimmermann, J. (2018). The time has come for dimensional personality disorder diagnosis. Personality and Mental Health, 12, 82-86. https://doi.org/10.1002/pmh.1408

Kenny, D., Kaniskan, B., \& McCoach, D. (2015). The performance of RMSEA in models with small degrees of freedom. Sociological Methods \& Research, 44, 486-507. https://doi.org/10.1177/0049124114543236

Krueger, R. F., Derringer, J., Markon, K. E., Watson, D., \& Skodol, A. E. (2013). The Personality Inventory for DSM-5 Brief Form (PID-5-BF). Manuscript in preparation.

Muthén, B. O. (1998-2004). Mplus technical appendices. Los Angeles, CA: Muthén \& Muthén.

Saris, W. E., \& Gallhofer, I. N. (2007). Design, evaluation, and analysis of questionnaires for survey research. Hoboken, NJ: Wiley.

Schermelleh-Engel, K., Moosbrugger, H., \& Müller, H. (2003). Evaluating the fit of structural equation models: Tests of significance and descriptive goodness-of-fit measures. Methods of Psychological Research, 8, 23-74.

Stoyanov, D., Machamer, P. K., \& Schaffner, K. F. (2012). Rendering clinical psychology an evidencebased scientific discipline: A case study. Journal of Evaluation in Clinical Practice, 18, 149-154. https:// doi.org/10.1111/j.1365-2753.2011.01795.x

Strus, W., Rowiński, T., Cieciuch, J., Kowalska-Dąbrowska, M., Czuma, I., \& Żechowski, C. (2017). The pathological big five: An attempt to build a bridge between the psychiatric classification of personality disorders and the trait model of normal personality. Roczniki Psychologiczne, 20, 451472. https://doi.org/10.18290/rpsych.2017.20.2-6en

Tomko, R. L., Trull, T. J., Wood, P. K., \& Sher, K. J. (2014). Characteristics of borderline personality disorder in a community sample: Comorbidity, treatment utilization, and general functioning. Journal of Personality Disorders, 28, 734-750. https://doi. org/10.1521/pedi_2012_26_093

Trull, T. J., Distel, M., \& Carpenter, R. (2011). DSM-5 borderline personality disorder: At the border between a dimensional and a categorical view. Current Psychiatry Reports, 13, 43-49. https://doi. org/10.1007/s11920-010-0170-2

Trull, T. J., \& Durrett, C. A. (2005). Categorical and dimensional models of personality disorder. Annual Review of Clinical Psychology, 1, 355-380. https:// doi.org/10.1146/annurev.clinpsy.1.102803.144009

Trull, T. J., Scheiderer, E. M., \& Tomko, R. L. (2012). Axis II comorbidity. In T. A. Widiger (Ed.), The $\mathrm{Ox}^{-}$ ford handbook of personality disorders (pp. 219236). New York, NY: Oxford University Press.
Widiger, T. A., \& Trull, T. J. (2007). Plate tectonics in the classification of personality disorder: Shifting to a dimensional model. American Psychologist, 62, 71-83. https://doi.org/10.1037/0003-066x.62.2.71

Zanarini, M. C., Vujanovic, A. A., Parachini, E. A., Boulanger, J. L., Frankenburg, F. R., \& Hennen, J. (2003). A screening measure for BPD: The McLean Screening Instrument for Borderline Personality Disorder (MSI-BPD). Journal of Personality Disorders, 17, 568-573. https://doi.org/10.1521/pedi.17.6.568.25355 